\title{
ÜBER GEWÖHNLICHE DIFFERENTIALUNGLEICHUNGEN ZWEITER ORDNUNG
}

\author{
ROLAND LEMMERT
}

\begin{abstract}
A theorem about the separation of sub- and superfunctions $v$ and $w$ by solutions of an ordinary differential equation of second order is proved, where $v>w$ throughout the given interval. Examples show that the condition imposed on the right side $f$ of the equation is sharp, and that an analogous theorem is not true for Laplace's equation, even in the case $f \equiv 0, v$ sub- and $w$ superharmonic.
\end{abstract}

1. Von Nagumo [2] stammt der folgende

SATZ 1. Es seien $v, w \in C^{2}(a, b) \cap C[a, b], v<w$ auf $[a, b]$. Die Funktion $f$ sei auf $M=\{(t, x): a \leqslant t \leqslant b, v(t) \leqslant x \leqslant w(t)\}$ stetig, und es gelte

$$
\begin{aligned}
& v^{\prime \prime}(t) \geqslant f(t, v), \quad t \in(a, b), \\
& w^{\prime \prime}(t) \leqslant f(t, w), \quad t \in(a, b) .
\end{aligned}
$$

Dann besitzt die Randwertaufgabe

$$
\begin{gathered}
u^{\prime \prime}(t)=f(t, u), \\
u(a)=u_{0}, \quad u(b)=u_{1}
\end{gathered}
$$

(mit $u_{0} \in[v(a), w(a)], u_{1} \in[v(b), w(b)]$ ) wenigstens eine Lösung $u$, die in $M$ verläuft.

Der triviale Sonderfall $f \equiv 0$ besagt dabei, daß zwischen einer konvexen Funktion $v$ und einer konkaven Funktion $w$ (mit $v<w$ ) stets eine lineare Funktion $u$ verläuft; dabei dürfen die Randwerte $u(a)$ bzw. $u(b)$ zwischen $v(a)$ und $w(a)$ bzw. $v(b)$ und $w(b)$ beliebig vorgeschrieben werden.

Kehrt man nun in (1), (2) die Ungleichungszeichen um, ist also $v$ konkav und $w$ konvex (mit $v \leqslant w$ ), so gibt es bekanntlich ebenfalls eine lineare Funktion $u$ zwischen $v$ und $w$; allerdings darf man in diesem Fall für $u$ keine Randwerte mehr vorschreiben.

Es wird im folgenden eine explizite Bedingung (5) an $f$ angegeben, so da $\beta$ eine entsprechende Aussage auch für Lösungen der Gleichung (3) gilt; um den Fall lediglich stetiger Funktionen $v$ und $w$ einzuschließen, werden dabei in (1) und (2) die zweiten Ableitungen also zweite Schwarzsche Derivierte aufgefaßt.

Schrader [3, Theorem 2.2] bewies eine solche Aussage für den Fall, daß $v, w \in$ $C^{2}[a, b]$ sind, mit Hilfe der Theorie der Unterfunktionen und Unterlösungen von Jackson (vgl. die bei Schrader angegebene Literatur); dabei darf $f$ noch von $u^{\prime}$

Received by the editors November 3, 1980.

1980 Mathematics Subject Classification. Primary 34A40, 26A51.

Key words and phrases. Generalized convex functions, separation theorem.

(c) 1981 American Mathematical Society 0002-9939/81/0000-0561/\$02.25 
abhängen, und es wird unter anderem vorausgesetzt, daß alle Lösungen der Anfangswertprobleme

$$
u^{\prime \prime}=f\left(t, u, u^{\prime}\right), \quad u\left(t_{0}\right)=u_{0}, \quad u^{\prime}\left(t_{0}\right)=m_{0}
$$

$\left(t_{0} \in[a, b], u_{0}, m_{0} \in \mathbf{R}\right)$ stets auf $[a, b]$ existieren. Diese Voraussetzung erfaßt etwa den Fall $f\left(t, u, u^{\prime}\right)=8 u^{3}$ nicht $\left(a=t_{0}=0, b=1, u_{0}=1, m_{0}=2\right.$ führt auf $u(t)=$ $\left.(1-2 t)^{-1}\right)$, der jedoch in unserer Bedingung an $f$ enthalten ist.

2. Für stetige Funktionen $\phi$ bezeichne $\bar{D}^{2} \phi$ bzw. $\underline{D}^{2} \phi$ die obere bzw. untere (zweite) Schwarzsche Derivierte, also mit $\Delta_{h} \phi(t)=\phi(t+h)-2 \phi(t)+\phi(t-h)$

$$
\bar{D}^{2} \phi(t)=\limsup _{h \rightarrow 0} \frac{\Delta_{h} \phi(t)}{h^{2}}, \quad \underline{D}^{2} \phi(t)=\liminf _{h \rightarrow 0} \frac{\Delta_{h} \phi(t)}{h^{2}}
$$

(vgl. etwa Zygmund [4]). $D^{+}, D_{+}, D^{-}, D_{-}$sind die vier Dini-Derivierten. Bekanntlich ist eine auf $(a, b)$ stetige Funktion $\phi$ genau dann konvex, wenn $\bar{D}^{2} \phi \geqslant 0$ auf $(a, b)$ gilt.

Definition 1. Die auf $(0,1)$ stetige Funktion $l$ gehört zu $E$, wenn eine Funktion $\psi \in C^{2}(0,1)$ existiert mit $\psi(t)>0, t \in(0,1)$ und $\psi^{\prime \prime}+l(t) \psi=0, t \in(0,1)$.

Ist $l \in E$, so ist bekanntlich für jedes $r \in C\left[t_{0}, t_{1}\right]$ das Randwertproblem

$$
\begin{aligned}
u^{\prime \prime}+l(t) u & =r(t), \quad t \in\left[t_{0}, t_{1}\right] \subset(0,1), \\
u\left(t_{0}\right) & =u_{0}, \quad u\left(t_{1}\right)=u_{1}
\end{aligned}
$$

eindeutig lösbar.

HilfsSATZ 1. Ist $f:(0,1) \times \mathbf{R} \rightarrow \mathbf{R}$ stetig und gilt

$$
f(t, x)-f(t, \bar{x}) \geqslant-l(t)(x-\bar{x}) \text { für } t \in(0,1), \quad x \geqslant \bar{x},
$$

mit $l \in E$, so besitzt für $0<t_{0}<t_{1}<1$ und jedes Paar $u_{0}, u_{1} \in \mathbf{R}$ die Gleichung (3) mit den Randbedingungen $u\left(t_{0}\right)=u_{0}, u\left(t_{1}\right)=u_{1}$ genau eine Lösung.

Der Beweis zu Hilfssatz 1 läßt sich mit Satz 1 führen: Geeignet gewählte Lösungen $v, w$ von (*) erfüllen die Ungleichungen (1) bzw. (2) auf $\left(t_{0}, t_{1}\right)$. Die Eindeutigkeitsaussage ergibt sich aus dem folgenden Hilfssatz.

HilfsSATZ 2. Ist $\phi$ auf $\left[t_{0}, t_{1}\right] \subset(0,1)$ stetig und gilt mit $l \in E$

$$
\begin{gathered}
\bar{D}^{2} \phi+l(t) \phi \geqslant 0, \quad t \in\left[t_{0}, t_{1}\right], \\
\phi\left(t_{0}\right) \leqslant 0, \quad \phi\left(t_{1}\right) \leqslant 0,
\end{gathered}
$$

so ist $\phi \leqslant 0$ auf $\left[t_{0}, t_{1}\right]$.

BeweIs. Wegen $l \in E$ gibt es ein $\psi \in C^{2}(0,1)$ mit $\psi>0$ auf $(0,1)$ und $\psi^{\prime \prime}+$ $l(t) \psi=0$. Für hinreichend kleines $\varepsilon>0$ besitzt deshalb das Problem

$$
u^{\prime \prime}+l(t) u=-\varepsilon, \quad u\left(t_{0}\right)=\psi\left(t_{0}\right), \quad u\left(t_{1}\right)=\psi\left(t_{1}\right)
$$

genau eine positive Lösung $\psi_{\varepsilon}$.

Ist nun $\phi>0$ für ein $t \in\left(t_{0}, t_{1}\right)$, so setzen wir

$$
\alpha_{0}=\inf \left\{\alpha>0: \alpha \psi_{\varepsilon}(t)>\phi(t), t \in\left[t_{0}, t_{1}\right]\right\} \text {. }
$$


Nach Annahme ist $\alpha_{0}>0$, und es existiert $\tau \in\left(t_{0}, t_{1}\right)$ mit $\alpha_{0} \psi_{e}(\tau)=\phi(\tau)>0$. Wegen $\alpha_{0} \psi_{\varepsilon}(t) \geqslant \phi(t)$ folgt $\alpha_{0} \psi_{e}^{\prime \prime}(\tau) \geqslant \bar{D}^{2} \phi(\tau)$ im Widerspruch zu

$$
\alpha_{0} \psi_{\varepsilon}^{\prime \prime}(\tau)=-\alpha_{0} l(\tau) \psi_{e}(\tau)-\alpha_{0} \varepsilon<-l(\tau) \phi(\tau)<\bar{D}^{2} \phi(\tau)
$$

nach (6).

Bemerkung. Aus Hilfssatz 2 folgt die Richtigkeit der Aussage (mit $l \in E$, $\left.\left[t_{0}, t_{1}\right] \subset(0,1)\right):$ Aus

$$
\bar{D}^{2} \phi+l \phi \geqslant u^{\prime \prime}+l u, \quad \phi\left(t_{0}\right)<u\left(t_{0}\right), \quad \phi\left(t_{1}\right)<u\left(t_{1}\right)
$$

folgt $\phi \leqslant u$ auf $\left[t_{0}, t_{1}\right]$.

HilfsSATZ 3. Ist $\phi$ auf $\left[t_{0}, t_{1}\right] \subset(0,1)$ stetig und gilt mit $l \in E$

$$
\bar{D}^{2} \phi+l \phi>0, \quad \phi\left(t_{0}\right)=0, \quad D^{+} \phi\left(t_{0}\right)>0,
$$

so ist $\phi>0$ auf $\left[t_{0}, t_{1}\right]$.

BEWEIS. Ist $\phi(\tau)<0$ für ein $\tau \in\left(t_{0}, t_{1}\right)$, so lösen wir

$$
\begin{array}{cc}
z^{\prime \prime}+l z=0 & \text { in }\left[t_{0}, \tau\right], \\
z\left(t_{0}\right)=0, & z(\tau)=\phi(\tau) .
\end{array}
$$

Es folgt leicht $\mathrm{z}^{\prime}\left(\mathrm{t}_{0}\right)<0$.

Aus der Bemerkung nach Hilfssatz 2 folgt $\phi(t)<z(t)$ in $\left[t_{0}, \tau\right]$, also wegen $\phi\left(t_{0}\right)=z\left(t_{0}\right)=0, D^{+} \phi\left(t_{0}\right)<z^{\prime}\left(t_{0}\right)<0$ im Widerspruch zur Voraussetzung.

BEMERKUNG. Eine entsprechende Aussage gilt für Lösungen von $\bar{D}^{2} \phi+l \phi \geqslant 0$, $\phi\left(t_{1}\right)=0, D_{-} \phi\left(t_{1}\right)<0$.

HilfsSATZ 4. Gilt für die stetige Funktion v

$$
\bar{D}^{2} v>h, \quad t \in(0,1),
$$

mit einer in $(0,1)$ stetigen Funktion $h$, so ist $v$ in $(0,1)$ rechts- und linksseitig differenzierbar; diese Ableitungen sind lokal beschränkt, und es gilt $\underline{D}^{2} v \geqslant h$ in $(0,1)$.

BeweIs. Wir wählen $H \in C^{2}(0,1)$ mit $H^{\prime \prime}=h$. Wegen $\bar{D}^{2}(v-H)=\bar{D}^{2} v-$ $H^{\prime \prime} \geqslant 0$ ist $v-H$ auf $(0,1)$ stetig und konvex, also rechts- und linksseitig differenzierbar mit lokal beschränkten Ableitungen. Damit besitzt auch $v$ diese Eigenschaften. Weiter gilt $\underline{D}^{2}(v-H)>0$, also wegen $\underline{D}^{2}(v-H)=\underline{D}^{2} v-H^{\prime \prime}$ $\operatorname{sogar} \underline{D}^{2} v>h$.

3. SATZ 2. Es seien $v, w \in C(0,1)$, es gelte $v<w$ und $f: M \rightarrow \mathbf{R}$ sei stetig $(M=\{(t, x): 0<t<1, v(t) \leqslant x \leqslant w(t)\})$. Gilt

$$
\underline{D}^{2} v<f(t, v), \quad \bar{D}^{2} w \geqslant f(t, w) \text { auf }(0,1)
$$

und erfüllt $f$ die Bedingung (5) mit $l \in E$, so existiert eine Lösung $u$ von (3), die in $M$ verläuft.

BEweIS. Wir beweisen Satz 2 zunächst für ein beliebiges Intervall $\left[t_{0}, t_{1}\right] \subseteq(0,1)$. Es existiere $\tau_{0} \in\left[t_{0}, t_{1}\right]$ mit $v\left(\tau_{0}\right)=w\left(\tau_{0}\right)$. (Der Fall $v(t)<w(t)$ auf $\left[t_{0}, t_{1}\right]$ wird anschließend auf diesen zurückgeführt.) Aus (8) in Verbindung mit Hilfssatz 4 folgt leicht $-\infty<w_{-}^{\prime} \leqslant w_{+}^{\prime}<\infty$ und $-\infty<v_{+}^{\prime} \leqslant v_{-}^{\prime}<\infty$, jeweils an der Stelle $\tau_{0}$. 
Weiter gelten die Ungleichungen $v_{+}^{\prime}\left(\tau_{0}\right)<w_{+}^{\prime}\left(\tau_{0}\right), v_{-}^{\prime}\left(\tau_{0}\right)>w_{-}^{\prime}\left(\tau_{0}\right)$. Damit gilt $\left[w_{-}^{\prime}, w_{+}^{\prime}\right] \cap\left[v_{+}^{\prime}, v_{-}^{\prime}\right] \neq \varnothing$, und diese Intervalle sind kompakt. Also existiert $m \in \mathbf{R}$ mit

$$
v_{+}^{\prime}\left(\tau_{0}\right)<m<w_{+}^{\prime}\left(\tau_{0}\right), \quad w_{-}^{\prime}\left(\tau_{0}\right)<m<v_{-}^{\prime}\left(\tau_{0}\right) .
$$

Nun denken wir uns $f$ auf $\left[t_{0}, t_{1}\right] \times \mathbf{R}$ durch

$$
\tilde{f}(t, x)=\left\{\begin{array}{l}
f(t, w(t)) \quad \text { für } x>w(t), \\
f(t, x) \text { für } v(t)<x \leqslant w(t), \\
f(t, v(t))+l(t)(v(t)-x) \text { für } x<v(t)
\end{array}\right.
$$

fortgesetzt, lösen

$$
u^{\prime \prime}(t)=\tilde{f}(t, u(t)), \quad t \in\left[t_{0}, t_{1}\right], \quad u\left(t_{0}\right)=v\left(t_{0}\right)=w\left(t_{0}\right), \quad u^{\prime}\left(t_{0}\right)=m
$$

und bezeichnen irgendeine Lösung mit $\bar{u}$. (Wegen der Eigenschaften von $\tilde{f}$ kann jede Lösung von (10) auf $\left[t_{0}, t_{1}\right]$ fortgesetzt werden.)

Wir beweisen zunächst $\bar{u}(t)<w(t), t \in\left[t_{0}, t_{1}\right]$. Wäre $\bar{u}\left(\tau_{1}\right)>w\left(\tau_{1}\right)$ etwa für $\tau_{1}>\tau_{0} \geqslant t_{0}$, so folgt $\bar{u}(t)>w(t)$ auf einem Intervall $\left(\tau_{0}^{\prime}, \tau_{1}\right]$ mit $\tau_{0}^{\prime}>\tau_{0}$ sowie $\bar{u}\left(\tau_{0}^{\prime}\right)=w\left(\tau_{0}^{\prime}\right)$. Auf diesem Intervall folgt dann für $d(t)=w(t)-\bar{u}(t)$

$$
\bar{D}^{2} d(t)=\bar{D}^{2} w(t)-\bar{u}^{\prime \prime}(t)>f(t, w(t))-\tilde{f}(t, \bar{u}(t))=0
$$

daß also $d$ auf $\left[\tau_{0}^{\prime}, \tau_{1}\right]$ konvex ist. Wegen $d\left(\tau_{0}^{\prime}\right)=0, d\left(\tau_{1}\right)<0$ folgt $d_{+}^{\prime}\left(\tau_{0}^{\prime}\right)<0$, also $w_{+}^{\prime}\left(\tau_{0}^{\prime}\right)<\bar{u}^{\prime}\left(\tau_{0}^{\prime}\right)$, also insbesondere $\tau_{0}^{\prime}>\tau_{0}$. Weiter gibt es $t \in\left[\tau_{0}, \tau_{0}^{\prime}\right]$ mit $\bar{u}(t)<$ $w(t)$ (da anderenfalls $\bar{u}^{\prime}\left(\tau_{0}^{\prime}\right) \leqslant w_{-}^{\prime}\left(\tau_{0}^{\prime}\right) \leqslant w_{+}^{\prime}\left(\tau_{0}^{\prime}\right)$ wäre), also jedenfalls ein Intervall $\left[s_{0}, s_{1}\right] \subseteq\left[\tau_{0}, \tau_{0}^{\prime}\right]$ mit $\bar{u}\left(s_{0}\right)=w\left(s_{0}\right), \bar{u}\left(s_{1}\right)=w\left(s_{1}\right), \bar{u}(t)<w(t)$ in $\left(s_{0}, s_{1}\right)$. In $\left(s_{0}, s_{1}\right)$ ist dann $\bar{D}^{2} d(t)=\bar{D}^{2} w(t)-\bar{u}^{\prime \prime}(t) \geqslant f(t, w)-f(t, \vec{u})>-l(t) d(t)$, im Widerspruch zu Hilfssatz 2.

Den Fall $\bar{u}(t)>w(t)$ für ein $t<\tau_{0}$ widerlegt man ebenso.

Schließlich zeigen wir $\bar{u}(t) \geqslant v(t), t_{0} \leqslant t \leqslant t_{1}$. Dazu bemerken wir, daß aufgrund der Definition von $\tilde{f}$

$$
\bar{D}^{2}(\bar{u}-v)=\bar{u}^{\prime \prime}-\underline{D}^{2} v>\tilde{f}(t, \bar{u})-f(t, v)>-l(t)(\bar{u}-v)
$$

in $\left[t_{0}, t_{1}\right]$ gilt. Hilfssatz 3 liefert $\bar{u}>v$ auf $\left[t_{0}, t_{1}\right]$. Damit ist Satz 2 für $\left[t_{0}, t_{1}\right]$ im Fall $\min _{t \in\left[t_{0}, t_{1}\right]}(w-v)=0$ bewiesen.

Ist $\min _{t \in\left[t_{0}, t_{1}\right]}(w-v)>0$, so setzen wir (mit $\psi$ aus der Definition 1)

$$
\alpha_{0}=\max \left\{\alpha>0: v(t)+\alpha \psi(t) \leqslant w(t), t \in\left[t_{0}, t_{1}\right]\right\} \text {. }
$$

Für $\bar{v}(t)=v(t)+\alpha_{0} \psi(t)$ gilt dann

$$
\begin{aligned}
\underline{D}^{2} \bar{v} & =\underline{D}^{2} v+\alpha_{0} \psi^{\prime \prime}(t) \\
& \leqslant f(t, v)-\alpha_{0} l(t) \psi(t) \leqslant f(t, \bar{v}), \quad t \in\left(t_{0}, t_{1}\right), \\
\bar{v} & \leqslant w \text { auf }\left[t_{0}, t_{1}\right],
\end{aligned}
$$

und es gibt $\tau_{0} \in\left[t_{0}, t_{1}\right]$ mit $\bar{v}\left(\tau_{0}\right)=w\left(\tau_{0}\right)$.

Nach dem oben Bewiesenen gibt es eine Lösung $\bar{u}$ auf $\left[t_{0}, t_{1}\right]$ mit $w(t)>u(t)>$ $\bar{v}(t)>v(t)$. Den allgemeinen Fall erhält man nun, indem man für $n \in \mathbf{N}$ auf den Intervallen $[1 / n, 1-1 / n]$ jeweils eine Lösung $u_{n}$ nach dem bisher Bewiesenen auswählt; die Folge $\left(u_{n}\right)$ besitzt eine in $(0,1)$ lokal gleichmäßig konvergente 
Teilfolge, und die Grenzfunktion $u$ ist eine Lösung von (3) für $t \in(0,1)$ mit $v<u<w, t \in(0,1)$.

BemerkUNG. In der Sprechweise von Beckenbach [1] ließe sich Satz 2 so formulieren: Gilt (5) für $f$, ist $v<w$ und ist $v$ bzw. $w$ im verallgemeinerten Sinn konkav bzw. konvex auf $(0,1)$, so lassen sich $v$ und $w$ durch eine Lösung von (3) trennen.

4. Wir zeigen anhand eines Beispiels, daß die Bedingung (5) für $f$ in Satz 2 scharf ist. Dazu sei $l(t) \equiv c^{2}$ mit $2 \pi>c>\pi$ und $f(t, x)=-c^{2} x+e^{-\alpha t}, t \in[0,1], x \in \mathbf{R}$, $\alpha \in \mathbf{R}$. Die Funktionen $v \equiv 0$ und $w=\frac{1}{2} t^{2}$ genügen der Voraussetzung von Satz 2 . Existiert $u$ mit den in Satz 2 angegebenen Eigenschaften, so ist $u(0)=u^{\prime}(0)=0$, also

$$
u(t)=\frac{1}{c^{2}+\alpha^{2}}\left(e^{-\alpha t}-\cos c t+\frac{\alpha}{c} \sin c t\right) .
$$

Insbesondere ist $u(1)=\left(1 / c^{2}+\alpha^{2}\right)\left(e^{-\alpha}-\cos c+(\alpha / c) \sin c\right)<0$ für hinreichend großes $\alpha$ im Widerspruch zu $u>0$.

Eine Satz 2 entsprechende Aussage etwa für elliptische Differentialgleichungen ist i.a. sogar für $f \equiv 0$ falsch. Dazu betrachten wir in der $x-y$-Ebene.

$$
\begin{aligned}
& v(x, y)=\left\{\begin{array}{l}
-\log \left[(x-2)^{2}+y^{2}\right], \quad x \leqslant 0, y \in \mathbf{R}, \\
\min \left\{-\log \left[(x-2)^{2}+y^{2}\right], \log \left[(x+2)^{2}+y^{2}\right]\right\},
\end{array} \quad x>0, y \in \mathbf{R},\right. \\
& w(x, y)= \begin{cases}\log \left[(x+2)^{2}+y^{2}\right], \quad x \geqslant 0, y \in \mathbf{R}, \\
\max \left\{-\log \left[(x-2)^{2}+y^{2}\right], \log \left[(x+2)^{2}+y^{2}\right]\right\}, & x<0, y \in \mathbf{R} .\end{cases}
\end{aligned}
$$

$v$ und $w$ sind etwa in $D=\left\{x^{2}+y^{2}<9\right\}$ stetig, $v$ ist superharmonisch, $w$ ist subharmonisch, es gilt $v \leqslant w$ und $v$ stimmt mit $w$ auf einer Umgebung von $(-2,0)$ und auf einer Umgebung von $(2,0)$ überein. Mit Hilfe des Identitätssatzes für harmonische Funktionen überlegt man sich leicht, daß zwischen $v$ und $w$ keine in $D$ harmonische Funktion liegen kann. Durch geeignete Glättung von $v$ und $w$ kann man sogar $v, w \in C^{\infty}$ erreichen. In diesem Zusammenhang vergleich man auch das Beispiel bei Amann [SIAM Rev. 18, S.653], wo $f$ linear in $u$ vorausgesetzt ist und für $u$ Randwerte auf $\partial D$ vorgeschrieben werden.

Herrn Professor Dr. Peter Volkmann, der mich auf diesen Problemkreis aufmerksam gemacht hat, möchte ich an dieser Stelle herzlich danken.

\section{LITERATUR}

1. E. F. Beckenbach, Generalized convex functions, Bull. Amer. Math. Soc. 43 (1937), 363-371.

2. M. Nagumo, Uber die Differentialgleichung $y^{\prime \prime}=f\left(x, y, y^{\prime}\right)$, Proc. Phys. Math. Soc. Japan 19 (1937), 861-866.

3. K. W. Schrader, Differential inequalities for second and third-order equations, J. Differential Equations 25 (1977), 203-215.

4. A. Zygmund, Trigonometric series, vol. I, 2nd ed., Cambridge Univ. Press, New York, 1959.

Mathematisches Institut I, Universität Karlsruhe, 75 Karlsruhe, Federal Republic of GERMANY 